\title{
The application of a targeted periprostatic nerve block in transperineal template-guided prostate biopsies
}

\author{
Xue-Fei Ding, Yang Luan, Fei Wang, Yao-Zong Xu, Cheng-Hao Guo, Liang-Yong Zhu \\ Clinical Medical College, Yangzhou University, Yangzhou, China \\ Correspondence to: Yang Luan, MD. Clinical Medical College, Yangzhou University, 98 West Nantong Road, Yangzhou 225001, China. \\ Email: Luan_Y@163.com.
}

\begin{abstract}
Background: Our study aims to evaluate the anesthetic efficacy of multiparametric magnetic resonance imaging/transrectal ultrasound (mpMRI/TRUS) fusion-guided targeted periprostatic nerve block (PNB) for transperineal template-guided prostate biopsy (TTPB).

Methods: The patients who underwent mpMRI/TRUS fusion-guided prostate biopsy from May 2018 to March 2019 were randomized into two groups using a random number table. The intervention group $(n=47)$ and the control group $(n=45)$ received targeted PNB and traditional PNB, respectively. Visual analog scale (VAS) and visual numeric scale (VNS) scores were used to assess the patients' pain and quantify their satisfaction.

Results: The total detection rate for prostate cancer was $45.7 \%$, with a comparable positive rate between the intervention group (42.6\%) and the control group (48.9\%), which meant there was no significant difference between the groups $(\mathrm{P}=0.542)$. Patient age, prostate-specific antigen, prostate volume, suspicious lesions on mpMRI, number of cores, operation time, and biopsy time were comparable between the groups. The VAS scores during biopsy were significantly lower in the intervention group than in the control group [2 (1 to 3) vs. 2 (1 to 4), $\mathrm{P}=0.019$ ]. Conversely, the VNS scores during biopsy were higher in the intervention group [3 (2 to 4 ) vs. 3 ( 2 to 3 ), $\mathrm{P}=0.015]$. There were no significant differences in the pain scores or the satisfaction scores at $30 \mathrm{~min}$ after the procedure between the two groups. There were no significant differences between the groups for complications, such as hematuria, urinary retention, infection, hemospermia, and vasovagal reaction $(\mathrm{P}>0.05)$.
\end{abstract}

Conclusions: Targeted PNB significantly relieved the pain and did not increase the incidence of complications for patients when compared with traditional PNB.

Keywords: Magnetic resonance imaging (MRI); prostate; nerve block; biopsy

Submitted Feb 29, 2020. Accepted for publication Aug 04, 2020.

doi: 10.21037/qims-20-369

View this article at: http://dx.doi.org/10.21037/qims-20-369

\section{Introduction}

Early diagnosis and effective treatment of prostate cancer (PCa) are essential for prolonging survival time and improving the quality of life of affected patients (1). Transperineal template-guided prostate biopsy (TTPB) is one of the most effective procedures for the detection of prostate cancer $(2,3)$. However, the biopsy may cause more pain than that caused by transrectal puncture (4).
Approximately $20 \%$ of patients have rejected prostate biopsy without anesthesia (5). Therefore, adequate anesthesia is a prerequisite of performing prostate puncture (6).

Currently, periprostatic nerve block (PNB) is safe, easily performed, and highly effective, and it is widely considered the preferred method for minimizing insertion pain from the biopsy needle in the prostatic tissue $(7,8)$. Accurately locating the neurovascular bundle (NVB) is the crucial step of PNB. The current method of locating the NVB is 
to locate the prostate-bladder-seminal vesicle angle $(9,10)$. However, unlike other solid tumors, the growth of the $\mathrm{PCa}$ is irregular (11); the position of the angle easily changes, leading to location difficulty and reduced anesthetic efficacy. Also, the distribution of the NVB in some patients is mutated $(12,13)$. Recently, studies have shown that multiparametric magnetic resonance imaging (mpMRI) has a high value in the localization of NVB $(14,15)$. Therefore, this study combined mpMRI and transrectal ultrasound (TRUS) to locate the NVB for performing targeted PNB accurately, and made comparisons with traditional PNB to evaluate whether targeted PNB offers better anesthetic efficacy.

\section{Methods}

\section{Study population}

From May 2018 to March 2019, the patients who underwent mpMRI/TRUS fusion-guided prostate biopsy were recruited. Study inclusion criteria were one or more of the following: (I) digital rectal examination findings nodules; (II) mpMRI findings indicative of prostate cancer; (III) PSA 4-10 ng/mL with an abnormal free/total PSA or PSA density; (IV) PSA $>10 \mathrm{ng} / \mathrm{mL}$. Exclusion criteria were: $\mathrm{mpMRI}$ revealing no suspicious areas, chronic pelvic pain syndrome, chronic prostatitis, previous prostate biopsies, allergy to local anesthetic, bleeding disorder, rectal pathology, active urinary tract infection, and taking analgesic medications.

All patients underwent mpMRI with a 3.0-T MR scanner (Signa HDxt, GE Medical Systems, Milwaukee, WI, USA) using a 32-channel phased-array coil before mpMRI/TRUS fusion-guided biopsy. The sequences of mpMRI included T1-weighted images (T1WI), T2-weighted images (T2WI), dynamic contrast enhancement (DCE), diffusion-weighted imaging (DWI), and apparent diffusion coefficient (ADC). Two experienced radiologists reviewed all mpMRI scans without having seen any prior clinical information.

The radiologists graded each suspicious area according to the prostate imaging reporting and data system (PI-RADS) version 2 as follows: (I) clinically significant cancer is highly unlikely to be present; (II) clinically significant cancer is unlikely to be present; (III) the presence of clinically significant cancer is equivocal; (IV) clinically significant cancer is likely to be present; and (V) clinically significant cancer is highly likely to be present (16). Finally, patients with a PI-RADS score of 2-5 were deemed candidates for
mpMRI/TRUS fusion-guided biopsy. An independent researcher used Microsoft Excel 2010 and a random number table to allocate the patients into two groups randomly. The random number table was concealed until all cases were complete and taken for analysis. The intervention group received targeted $\mathrm{PNB}$, while the control group received traditional PNB. Ethics clearance was granted by the Clinical Medical College of the Yangzhou University (2018KY-032). Each patient gave written informed consent before undergoing a prostate biopsy.

The Epi Info ${ }^{\mathrm{TM}} 7$ (WHO, Geneva, Switzerland) program was used to calculate the sample size. The limits were set as $95 \% \mathrm{CI}, 5 \% \alpha$ error, $80 \%$ power, and $90 \%$ population. It was estimated that $\geq 36$ men would be needed for each of the two groups. And according to the predicted 20\%, clinical missed follow-up rate, $\geq 44$ patients in each group were required.

\section{mpMRI/TRUS image fusion}

Before the prostate biopsy, a complete blood count with differentiation, coagulation test, and a comprehensive metabolic panel was routinely performed. The patients were given oral intestinal clearance drugs one day before the operation and emptied their bowels on the morning of the operation.

All biopsies were conducted with a mpMRI/TRUS biopsy system (MIM Symphony, MIM Software, Cleveland, OH, USA), providing a real-time fusion of TRUS and mpMRI images to guide the biopsy needle using a transperineal approach. Firstly, the original data from the mpMRI in digital imaging and communications in medicine (DICOM) format were introduced into the image fusion system. Then, we marked the suspicious areas on the T2WI (Figure 1A); the NVB was also marked on T2WI in the intervention group (Figure 1A). Second, the virtual probe was placed in the rectum (approximately $3 \mathrm{~mm}$ from the posterior wall of the prostate). Next, the angle of the probe was adjusted until the base plane was aligned with the underlying prostate, and the virtual grid was adjusted to ensure that the prostate was in a suitable place. Finally, a biplanar TRUS probe (Flex Focus 1202 rectal ultrasound, BK, Naerum, Denmark) was inserted into the patient's rectum. The prostate base was used as the fiducial landmark for registering the MRI and ultrasound images. The reconstructed MRI axial images were synchronously displayed adjacent to the TRUS axial image on the same monitor (Figure 1B). Morphological MRI data, including 

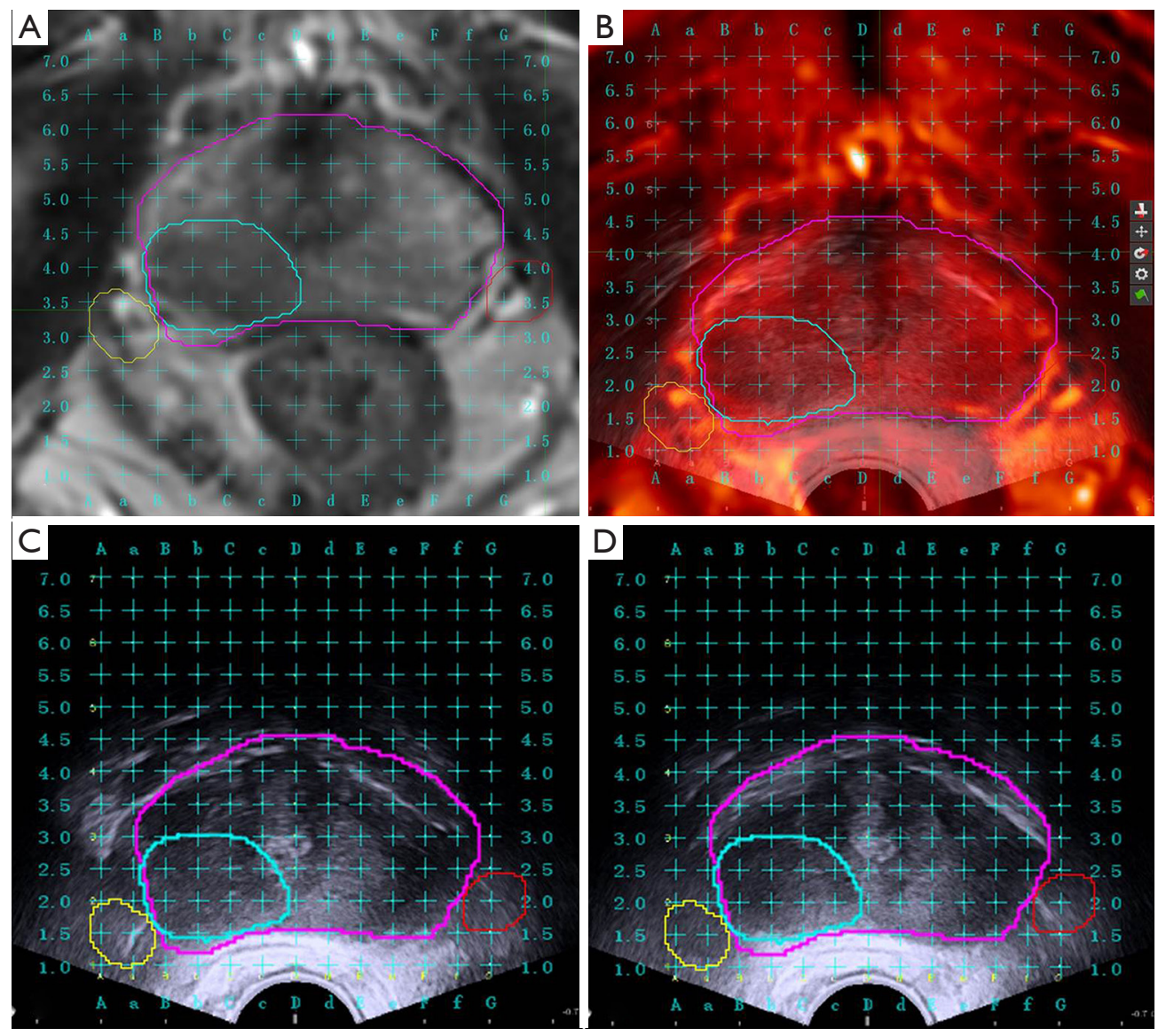

Figure $1 \mathrm{mpMRI}$ TRUS image fusion. (A) Multiple sequences of mpMRI, the regions of interest are delineated on T2WI. The prostate target is contoured in purple. The suspected area of prostate cancer target is contoured in blue. The bilateral NVB target is contoured in yellow and red, respectively. (B) The reconstructed MRI axial images are synchronously displayed adjacent to the TRUS axial image on the same monitor. With the brightness of MRI image adjusted, the MRI image with a red background can be seen behind the TRUS image, and the goodness of fit between the MRI and the TRUS image could be observed and adjusted. (C,D) Targeted PNB was performed under the guidance of the mpMRI/TRUS image fusion. Infiltrative anesthesia for NVB in yellow and red contours was performed, respectively. The bright white spots inside yellow (C) and red (D) contours were the needlepoint of the ANSll $0.5 \mathrm{~mm} \times 112 \mathrm{~mm}$ spinal needle.

the suspicious areas, were superimposed on the TRUS images in real-time to guide the PNB and the biopsy needle.

\section{Anesthesia}

(I) Perineal area skin infiltration anesthesia was performed with $10 \mathrm{ml}$ of $1 \%$ lidocaine, guided by intraoperative ultrasound. The scope of infiltration was $0.5 \mathrm{~cm}$ greater than the projection area of the prostate onto the perineal skin. (II) Infiltration anesthesia was then performed on the apex of the prostate. The syringe was inserted at the
$1,3,5,7,9$, and 11 o'clock positions of the prostate apex projected in the perineal skin. After, we injected $12 \mathrm{~mL}$ of $1 \%$ lidocaine ( $2 \mathrm{~mL}$ in each point) near the apex of the prostate. (III) For the intervention group, according to the position of bilateral NVB displayed in the fusion image, $5 \mathrm{~mL}$ of $1 \%$ lidocaine was injected using an ANSll $0.5 \mathrm{~mm} \times 112 \mathrm{~mm}$ spinal needle (Figure $1 C, D$ ). In the control group, traditional PNB was performed. Under the guidance of TRUS, a rotating probe was used to find the position of the blood vessel of NVB, the juncture of the location was deemed the prostate-bladder-seminal vesicle angle. Into this area, $5 \mathrm{~mL}$ of $1 \%$ lidocaine was injected using an ANSll 


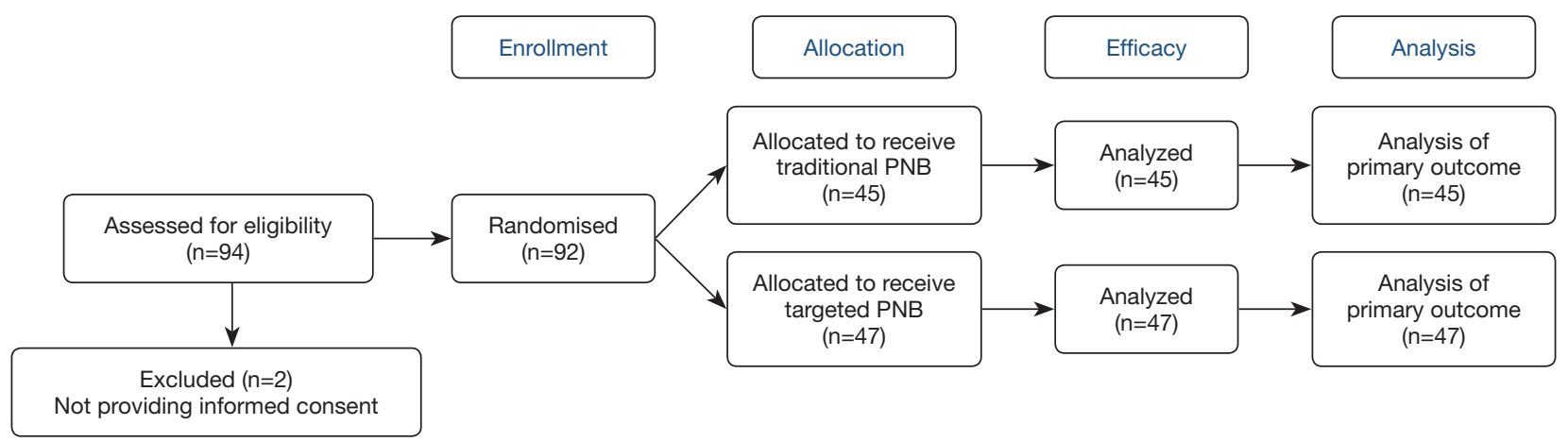

Figure 2 The flow diagram of the study according to the Consolidated Standards of Reporting Trials (CONSORT) guidelines.

$0.5 \mathrm{~mm} \times 112 \mathrm{~mm}$ spinal needle, all as described previously (17). This procedure was repeated bilaterally.

Following anesthesia, TTPB was performed for 5 minutes.

\section{Pain and complication assessment}

A visual analog scale (VAS; 0, none; 10, intolerable pain) was used to evaluate pain, and a visual numeric scale (VNS; 0 , terrible; 4 , perfect) was used to quantify their satisfaction; only whole numbers were accepted for both scale ratings $(18,19)$. An independent resident and the participants were blinded to the anesthesia method. The independent resident explained the VAS and VNS to the patients and requested them to rate their level of pain and satisfaction, respectively. The VAS and VNS were rated at two intervals. VAS-1 and VNS-1 were determined during the biopsy procedure, and VAS-2 and VNS-2 were determined 30 min after the procedure. The patients recorded the VAS and VNS scores without any other assistance.

Patients were given a nonvalidated, self-administered questionnaire about complications, such as hematuria, urinary retention, infection, hemospermia, and vasovagal reaction.

\section{Statistical analysis}

Statistical software SPSS (version 19.0; IBM Corp, Armonk, NY, USA) was used for data processing. Quantitative statistics were described using mean $\pm \mathrm{SD}$, and grade data were displayed as a median (interquartile range), whereas enumeration data were expressed as percentages. The Mann-Whitney $U$ test was used for pain and satisfaction score comparisons. The chi-squared $\left(\chi^{2}\right)$ test was used to compare the rates of complications. A two-tailed $\mathrm{P}<0.05$ indicated statistical significance.

\section{Results}

A total of 94 patients with suspected PCa underwent mpMRI/TRUS fusion-guided prostate biopsy. Of these, two patients did not provide consent, leaving 92 patients included in the analysis; there were 47 patients in the intervention group and 45 patients in the control group (Figure 2). The demographic characteristics of the cohort are described in Table 1 . In the intervention group, the mpMRI lesions per patient were $1.8 \pm 0.6$, and the number of biopsy cores was $3.6 \pm 1.3$. In the control group, the mpMRI lesions per patient and the number of biopsy cores were $2.0 \pm 0.8$ and $3.8 \pm 1.5$, respectively. There was no statistically significant difference in the mpMRI lesions per patient or the number of biopsy cores between the two groups ( $>>0.05)$. The operation time, including the mpMRI/TRUS fusion time, biopsy time, and anesthesia was $25.1 \pm 11.6 \mathrm{~min}$ in the intervention group and $22.5 \pm 10.2 \mathrm{~min}$ in the control group $(\mathrm{P}=0.253)$. The biopsy time from the beginning of the first needle to the end of the last needle was $2.7 \pm 1.1$ and $2.9 \pm 1.3$ in the intervention group and the control group, respectively $(\mathrm{P}=0.427)$. The positive rates of $\mathrm{PCa}$ in the intervention group and the control group were $42.6 \%$ $(20 / 47)$ and $48.9 \%(22 / 45)$, respectively $(P=0.542)$. The difference in age, PSA, and prostate volume between the groups was not significant $(\mathrm{P}>0.05)$.

In the intervention group and the control group, VAS-1 scores were 2 ( 1 to 3 ) and 2 ( 1 to 4 ), and VNS-1 scores were 3 ( 2 to 4 ) and 3 ( 2 to 3 ), respectively. The pain scores in the intervention group were significantly lower than in the control group $(\mathrm{P}=0.019)$. Satisfaction scores in the 
Table 1 Patient characteristics and summary of biopsy findings

\begin{tabular}{|c|c|c|c|}
\hline Characteristics & Intervention group & Control group & $P$ value \\
\hline Age (years) & $68.5 \pm 7.4$ & $67.9 \pm 8.5$ & 0.719 \\
\hline PSA ( $\mu \mathrm{g} / \mathrm{L})$ & $10.8 \pm 6.9$ & $12.1 \pm 7.3$ & 0.381 \\
\hline Prostate volume (mL) & $44.2 \pm 16.4$ & $42.5 \pm 17.9$ & 0.635 \\
\hline No. of cores & $3.6 \pm 1.3$ & $3.8 \pm 1.5$ & 0.495 \\
\hline Operation time (min) & $25.1 \pm 11.6$ & $22.5 \pm 10.2$ & 0.253 \\
\hline Biopsy time (min) & $2.7 \pm 1.1$ & $2.9 \pm 1.3$ & 0.427 \\
\hline Positive rate (\%) & 42.6 & 48.9 & 0.542 \\
\hline
\end{tabular}

Table 2 The VAS scores and VNS scores in the two groups

\begin{tabular}{lccccc}
\hline Groups & No. of men & VAS-1 & VAS-2 & VNS-1 & VNS-2 \\
\hline Intervention group & 47 & $2(1$ to 3$)$ & 0 (0 to 1) & $3(2$ to 4$)$ & $3(3$ to 4$)$ \\
Control group & 45 & $2(1$ to 4$)$ & $0(0$ to 1$)$ & $3(2$ to 3) & $3(3$ to 4$)$ \\
P value & & 0.019 & 0.140 & 0.015 & 0.274 \\
\hline
\end{tabular}

Table 3 Complications of the operation

\begin{tabular}{lccccc}
\hline Groups & Hematuria & Urinary retention & Infection & Hemospermia & Vasovagal reaction \\
\hline Intervention group & $14.9 \%(n=7)$ & $2.1 \%(n=1)$ & 0 & $0 \%(n=0)$ & $0.0 \%(n=0)$ \\
Control group & $11.1 \%(n=5)$ & $0 \%(n=0)$ & 0 & $2.2 \%(n=1)$ & $2.2 \%(n=1)$ \\
$P$ value & 0.590 & 1 & 1 & 0.489 & 0.489 \\
\hline
\end{tabular}

intervention group were significantly higher than in the control group $(\mathrm{P}=0.015)$. The VAS-2 scores were 0 ( 0 to 1$)$ and 0 ( 0 to 1$)$, and VNS-2 scores were 4 (3 to 4 ) and 3 ( 3 to 4 ) in the intervention group and the control group, respectively. There were no significant differences in pain scores or satisfaction scores at $30 \mathrm{~min}$ after the procedure among the two groups (Table 2).

There were five patients $(11.1 \%)$ with hematuria in the control group and seven patients $(14.9 \%)$ with hematuria in the intervention group after the puncture, and there was no statistically significant difference between the two groups $(\mathrm{P}=0.590)$. Urinary retention was observed in one patient $(2.1 \%)$ in the intervention group, and there was no statistically significant difference between the two groups $(\mathrm{P}=1)$. In the control group, one patient (2.2\%) developed hematospermia, which was fully resolved after conservative treatment. In the control group, one patient $(2.2 \%)$ presented with a vasovagal reaction, which improved after bed rest. Postoperative fever was not observed in either group (Table 3).

\section{Discussion}

The TTPB method has become one of the most commonly used for the diagnosis of $\mathrm{PCa}$. As an invasive procedure, it is associated with significant pain and discomfort. Currently, PNB is considered the 'gold standard' for pain control during prostate biopsy $(7,8)$. It was first reported in 1996 by Nash et al. (20) that PNB decreased pain during TRUSguided prostate biopsy. Conde Redondo et al. (21) found that bilateral PNB had a better anesthetic efficacy than oral morphine. A meta-analysis of 14 studies conducted by 
Hergan (22) showed that PNB was better than a placebo or no anesthesia. The most critical step in PNB is to locate the NVB. Currently, the most effective localization method is to find the blood vessels of the NVB by TRUS and locate the prostate nerve plexus with blood vessels, and the location used is the prostate-bladder-seminal vesicle angle (23). Therefore, irregular hyperplasia of the prostate causes this angular position to change, which may affect the efficacy of anesthesia. Also, some studies found that NVB distribution is variable in some patients, and not all the peripheral nerves of the prostate are located at that angle (24). Hence, there is a need for a method that is sensitive enough to accurately position the NVB, to improve the anesthetic efficacy of PNB.

With the advancement of technology, the mpMRI can now scan any plane and provide more accurate measurement tools, with good tissue and high spatial resolution (25). Wei et al. (15) found that MRI was advantageous in identifying the NVB. On the MRI, the prostate peripheral vessel and nerve formed quasi-circular low-signal structures, connected serially in grape-like structures, or fused into an arc structure which was distributed in the pelvic fascia on both sides of the prostate. Furthermore, Kwon et al. (14) found that T2WI was superior to T1WI for locating the NVB. Meanwhile, mpMRI/TRUS fusion navigation technology has become an accurate targeted biopsy method for suspicious lesions because mpMRI/TRUS can display real-time TRUS images and display MRI images of the same ultrasound section concurrently. This fusion significantly improved the positive rate of puncture, hence reducing the number of puncture needles required $(26,27)$. However, currently, spinal or even general are the predominant anesthesia methods used for mpMRI/TRUS fusion-guided prostate biopsy, these methods are timeconsuming, laborious, and present increased anesthesia risks $(28,29)$. There have been previous studies that applied PNB to mpMRI/TRUS fusion-guided prostate biopsy (30); however, only the traditional PNB was performed. Hence, our study aimed to achieve increased anesthetic efficacy when mpMRI/TRUS fusion-guided prostate biopsy was performed; targeted PNB was performed with accurate location enabled by fusing the image of NVB displayed on MRI with the TRUS image. Our study revealed that the pain scores in the intervention group were significantly lower than in the control group, and that satisfaction scores in the intervention group were significantly higher than in the control group. However, complications, including hematuria, urinary retention, infection, hemospermia, and vasovagal reaction, were not significantly different between the groups. Hence, targeted PNB provided improved anesthesia compared to traditional PNB and did not increase the incidence of complications. Moreover, targeted PNB enabled mpMRI/TRUS fusion-guided biopsy to be performed in the outpatient facility; conversely, lumbar or general anesthesia procedures need to be performed in the operating room.

Our study involved some limitations. In the process of registering MRI and TRUS images, prostate displacement and deformation are easily caused by changes in the patient's position, ultrasound probe diameter inconsistencies, and the MRI rectal coil; these variables lead to deviations in registration. Additionally, the corresponding TRUS images must be subjectively selected for registration to determine the level following MRI image selection of the marker point, which results in the error of image registration to a certain extent. Moreover, although VAS and VNS scores are relatively objective indicators of pain, assessment is based on patients' subjective sense of pain and is, therefore, lacking in objective quantitative indicators. Further research is needed to address these limitations.

\section{Conclusions}

The images of MRI are high resolution and have a large number of parameters; when combined with the real-time imaging of TRUS, the fusion of the two imaging techniques will improve the localization accuracy of the NVB. Targeted PNB, based on the fusion of MRI and TRUS images, can reduce the pain of puncture without increasing the incidence of complications.

\section{Acknowledgments}

Funding: This work was supported by the Jiangsu Provincial Commission of Health and Family Planning Research Project (no. H201550).

\section{Footnote}

Conflicts of Interest: All authors have completed the ICMJE uniform disclosure form (available at http://dx.doi. org/10.21037/qims-20-369). The authors have no conflicts of interest to declare

Ethical Statement: Ethics clearance was granted by the Clinical Medical College of the Yangzhou University 
(2018KY-032). Each patient gave written informed consent before undergoing a prostate biopsy.

Open Access Statement: This is an Open Access article distributed in accordance with the Creative Commons Attribution-NonCommercial-NoDerivs 4.0 International License (CC BY-NC-ND 4.0), which permits the noncommercial replication and distribution of the article with the strict proviso that no changes or edits are made and the original work is properly cited (including links to both the formal publication through the relevant DOI and the license). See: https://creativecommons.org/licenses/by-nc-nd/4.0/.

\section{References}

1. Li M, Chen T, Zhao W, Wei C, Li X, Duan S, Ji L, Lu Z, Shen J. Radiomics prediction model for the improved diagnosis of clinically significant prostate cancer on biparametric MRI. Quant Imaging Med Surg 2020;10:368-79.

2. Muthuveloe D, Telford R, Viney R, Patel P. The detection and upgrade rates of prostate adenocarcinoma following transperineal template-guided prostate biopsy - a tertiary referral centre experience. Cent European J Urol 2016;69:42-7.

3. Mai Z, Xiao Y, Yan W, Zhou Y, Zhou Z, Liang Z, Ji Z, Li H. Comparison of lesions detected and undetected by template-guided transperineal saturation prostate biopsy. BJU Int 2018;121:415-20.

4. Udeh EI, Amu OC, Nnabugwu, II, Ozoemena O. Transperineal versus transrectal prostate biopsy: our findings in a tertiary health institution. Niger J Clin Pract 2015;18:110-4.

5. Bingqian L, Peihuan L, Yudong $W$, Jinxing $W$, Zhiyong $W$. Intraprostatic local anesthesia with periprostatic nerve block for transrectal ultrasound guided prostate biopsy. J Urol 2009; 182:479-83; discussion 483-4.

6. Ukimura O, Coleman JA, de la Taille A, Emberton M, Epstein JI, Freedland SJ, Giannarini G, Kibel AS, Montironi R, Ploussard G, Roobol MJ, Scattoni V, Jones JS. Contemporary role of systematic prostate biopsies: indications, techniques, and implications for patient care. Eur Urol 2013;63:214-30.

7. Wang J, Wang L, Du Y, He D, Chen X, Li L, Nan X, Fan J. Addition of intrarectal local analgesia to periprostatic nerve blockimproves pain control for transrectal ultrasonography-guidedprostate biopsy: A systematic review and meta-analysis. Int J Urol 2015;22:62-8.
8. Otunctemur A, Dursun M, Besiroglu H, Can Polat E, Cakir SS, Ozbek E, Karadeniz T. The effectivity of periprostatic nerve blockade for the pain control during transrectal ultrasound guided prostate biopsy. Arch Ital Urol Androl 2013;85:69-72.

9. Atta H, Mostafa MF, Shalaby M. Which is better for pain reduction during transrectal ultrasound-guided biopsy of the prostate: Intravenous diazepam, local periprostatic nerve block, or combination? controlled randomized study. Saudi J Anaesth 2018;12:16-21.

10. Jindal T, Mukherjee S, Sinha RK, Kamal MR, Ghosh N, Saha B, Mitra N, Sharma PK, Mandal SN, Karmakar D. Transrectal ultrasonography (TRUS)-guided pelvic plexus block to reduce pain during prostate biopsy: a randomised controlled trial. BJU Int 2015;115:892-6.

11. Konyalioglu E, Tarhan H, Cakmak O, Pala EE, Zorlu F. Prostate cancer volume estimations based on transrectal ultrasonography-guided biopsy in order to predict clinically significant prostate cancer. Int Braz J Urol 2015;41:442-8.

12. Alsaid B, Karam I, Bessede T, Abdlsamad I, Uhl JF, Delmas V, Benoit G, Droupy S. Tridimensional computerassisted anatomic dissection of posterolateral prostatic neurovascular bundles. Eur Urol 2010;58:281-7.

13. Costello AJ, Dowdle BW, Namdarian B, Pedersen J, Murphy DG. Immunohistochemical study of the cavernous nerves in the periprostatic region. BJU Int 2011;107:1210-5.

14. Kwon T, Lee C, Jung J, Kim CS. Neurovascular bundle size measured on 3.0-T magnetic resonance imaging is associated with the recovery of erectile function after robot-assisted radical prostatectomy. Urol Oncol 2017;35:542.e11-7.

15. Wei S, Cheng F, Li H, Yu W, Rao T. The value of preoperative magnetic resonance imaging in detecting the distribution of neurovascular bundles before the nervesparing laproscopic radical prostatectomy. Chinese Journal of Urology, 2018;39:522-6.

16. Weinreb JC, Barentsz JO, Choyke PL, Cornud F, Haider MA, Macura KJ, Margolis D, Schnall MD, Shtern F, Tempany CM, Thoeny HC, Verma S. PI-RADS Prostate Imaging - Reporting and Data System: 2015, Version 2. Eur Urol 2016;69:16-40.

17. Luan Y, Huang TB, Gu X, Zhou GC, Lu SM, Tao HZ, Liu BD, Ding XF. Effect of prostate volume on the peripheral nerve block anesthesia in the prostate biopsy: A strobe-compliant study. Medicine (Baltimore) 2016;95:e4184. 
18. Tüfek I, Akpinar H, Atug F, Obek C, Esen HE, Keskin MS, Kural AR. The impact of local anesthetic volume and concentration on pain during prostate biopsy: a prospective randomized trial. J Endourol 2012;26:174-7.

19. Doğanca T, Savsin A, Erdogan S, Altindas F, Ozdemir F, Ekici B, Obek C. Procedural sedation and analgesia as an adjunct to periprostatic nerve block for prostate biopsy: A prospective randomized trial. J Clin Ultrasound 2015;43:288-94.

20. Nash PA, Bruce JE, Indudhara R, Shinohara K. Transrectal ultrasound guided prostatic nerve blockade eases systematic needle biopsy of the prostate. J Urol 1996;155:607-9.

21. Conde Redondo C, Alonso Fernandez D, Robles Samaniego A, Del Valle Gonzalez N, Castroviejo Royo F, Delgado Marcos C, Rodriguez Toves A, Martinez-Sagarra Oceja JM. TRUS-guided biopsy: comparison of two anesthetic methods. Actas Urol Esp 2006;30:134-8.

22. Hergan L, Kashefi C, Parsons JK. Local anesthetic reduces pain associated with transrectal ultrasound-guided prostate biopsy: a meta-analysis. Urology 2007;69:520-5.

23. Urabe F, Kimura T, Shimomura T, Onuma H, Yamamoto T, Sasaki H, Miki J, Kuruma H, Miki K, Egawa S. Prospective comparison of the efficacy of caudal versus periprostatic nerve block, both with intrarectal local anesthesia, during transrectal ultrasonography-guided prostatic needle biopsy. Scand J Urol 2017;51:245-50.

24. Sievert KD, Hennenlotter J, Dillenburg T, Toomey P, Wollner J, Zweers P, Pannek J, Andersson KE, Amend B. Extended periprostatic nerve distributions on the prostate surface confirmed using diffusion tensor imaging. BJU Int 2019;123:995-1004.

25. Xi Y, Liu A, Olumba F, Lawson P, Costa DN, Yuan Q, Khatri G, Yokoo T, Pedrosa I, Lenkinski RE. Low-to- high b value DWI ratio approaches in multiparametric MRI of the prostate: feasibility, optimal combination of $\mathrm{b}$ values, and comparison with ADC maps for the visual presentation of prostate cancer. Quant Imaging Med Surg 2018;8:557-67.

26. Baco E, Rud E, Eri LM, Moen G, Vlatkovic L, Svindland A, Eggesbo HB, Ukimura O. A Randomized Controlled Trial To Assess and Compare the Outcomes of Two-core Prostate Biopsy Guided by Fused Magnetic Resonance and Transrectal Ultrasound Images and Traditional 12-core Systematic Biopsy. Eur Urol 2016;69:149-56.

27. Tonttila PP, Lantto J, Paakko E, Piippo U, Kauppila S, Lammentausta E, Ohtonen P, Vaarala MH. Prebiopsy Multiparametric Magnetic Resonance Imaging for Prostate Cancer Diagnosis in Biopsy-naive Men with Suspected Prostate Cancer Based on Elevated Prostate-specific Antigen Values: Results from a Randomized Prospective Blinded Controlled Trial. Eur Urol 2016;69:419-25.

28. Bonekamp D, Schelb P, Wiesenfarth M, Kuder TA, Deister F, Stenzinger A, Nyarangi-Dix J, Rothke M, Hohenfellner M, Schlemmer HP, Radtke JP. Histopathological to multiparametric MRI spatial mapping of extended systematic sextant and MR/TRUS-fusion-targeted biopsy of the prostate. Eur Radiol 2019;29:1820-30.

29. Kosarek CD, Mahmoud AM, Eyzaguirre EJ, Shan Y, Walser EM, Horn GL, Williams SB. Initial series of magnetic resonance imaging (MRI)-fusion targeted prostate biopsy using the first transperineal targeted platform available in the USA. BJU Int 2018;122:909-12.

30. Robins D, Lipsky M, RoyChoudry A, Wenske S. Assessment of Discomfort and Pain in Patients Undergoing Fusion Magnetic Resonance Imaging-guided vs TRUS-guided Prostate Biopsy. Urology 2018;116:30-4.
Cite this article as: Ding XF, Luan Y, Wang F, Xu YZ, Guo CH, Zhu LY. The application of a targeted periprostatic nerve block in transperineal template-guided prostate biopsies. Quant Imaging Med Surg 2020;10(11):2125-2132. doi: 10.21037/qims-20-369 